\title{
Fluorescence imaging of mitochondrial responses to glucose challenge and probes in mitochondrial DNA-deficient osteosarcoma cells.
}

\author{
E Kohen*, JG Hirschberg**, C Örnek*, M Monti**, JP Berry*** and R LeBlanc*** \\ Departments of *Biology, **Physics, and ***Chemistry, University of Miami, Coral Gables, FL \\ 33124, USA
}

F. N. Ghadially has published an ultrastructural pathology of the cell and matrix which describes the morphological changes associated with a large number of diseases, genetic disorders and toxic effects[1]. We have attempted the counterpart of such studies with living cells, using fluorescence probing and imaging of cells' organelles. Fluorescence imaging of organelle interactions has been carried out using a variety of excitation and imaging filters. The method has been originally tried for mitochondrial visualization in a variety of cells including yeast, mastocytoma, transformed- keratinocytes and mitochondrial DNA deficient osteosarcoma [2]. Vital probes used included DASPMI, Mitotracker Green, TMRE for mitochondria, and quinacrine for lysosomes (Fig. 1).

The stages of mitochondrial NADH responses to glucose challenge are being evaluated in osteosarcoma (Fig. 2). Under glucose challenge, the mitochondria responded within seconds by increased NADH fluorescence which appeared in a few scattered locations and then spread rapidly in a few seconds with increased intensity to the rest of the mitochondria. Even though the quantum yield of NADH fluorescence is weak, it was possible to record the fluorescence (Fig. 3). This marks the osteosarcoma cell as an excellent example for further studies of mitochondrial metabolic activation.

Based on the demonstration by Gregorio Weber and Ronald Gemperlein, the scope of this study can be broadened by using the excitation option of fluorescence imaging $[3,4]$. In principle, excitation and emission filters can be replaced by various forms of double beam interferometry in conjunction with Fourier analysis [5]. A recently constructed "Pentaferometer" appears to be quite suitable for insertion on the excitation or emission side, or on both sides [6]. The method is deemed suitable for extension to neuroblastoma, lysosomal disease cells and senescent cells in diagnostics or drug trials. Complementation is considered by photoacoustic spectroscopy.

\section{References}

[1] F.N. Ghadially, Ultrastructural Pathology of Cell and Matrix, Vols 1 and 2, Butterworths, London, 1988.

[2] E. Kohen et al., Cancer Detection and Prevention, Abstract 382 (2002 Symposium Volume), 2002.

[3] G. Weber, Nature 190 (1961) 27.

[4] R. Gemperlein, Doc. Opthalmol Proc. Ser. 33 (1978) 265.

[5] J.G. Hirschberg and E. Kohen, Appl. Optics, 38 (1999) 136.

[6] C. Rothmann and Z. Malik et al. in Applications of Optical engineering to the Study of

Cellular Pathology (Ed. E. Kohen and J.G. Hirschberg) Research Signpost, Trivandrum, 1999. 


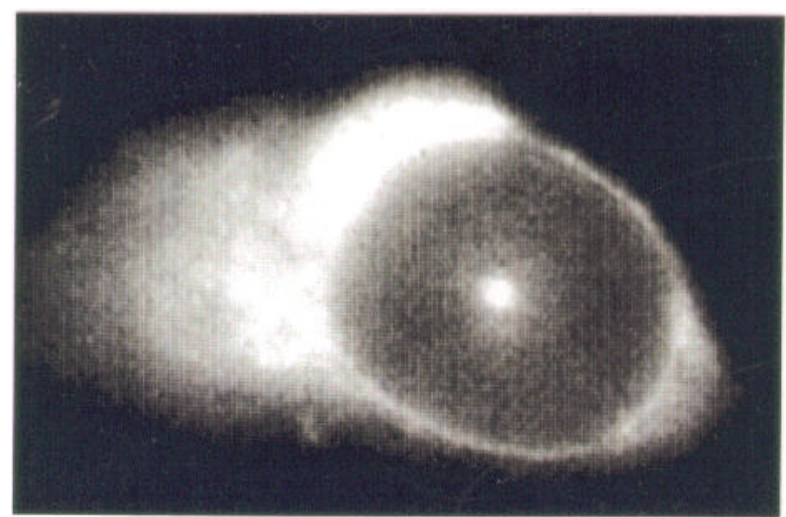

Figure 1: Osteosarcoma mutant 136 $\square 0$ treated with Mitotracker Green.
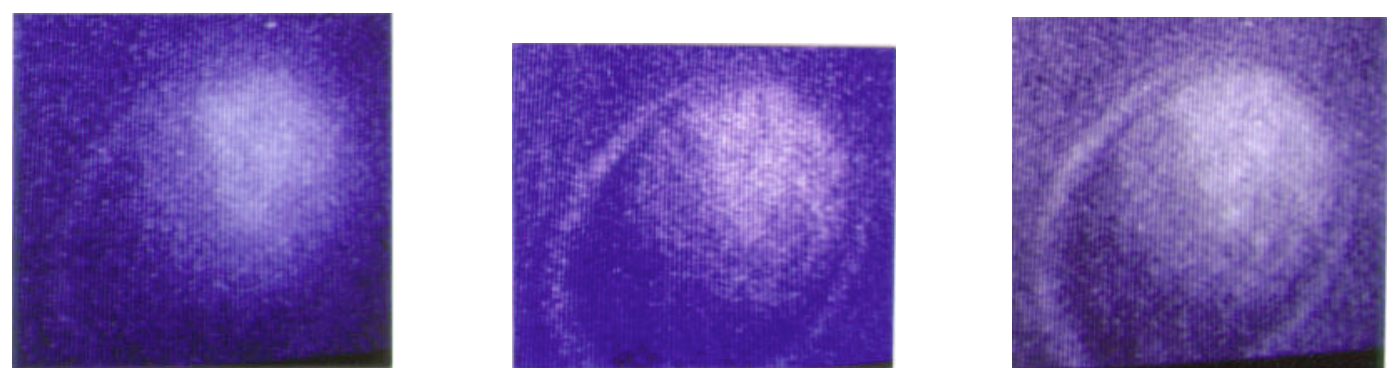

Figure 2: Weak NADH fluorescence response of osteosarcoma $136 \square 0$ mitochondria to glucose challenge. A. before glucose; B. immediately after; C. about 60 seconds after. Contrast with high quantum yield of emission in Figure 1.

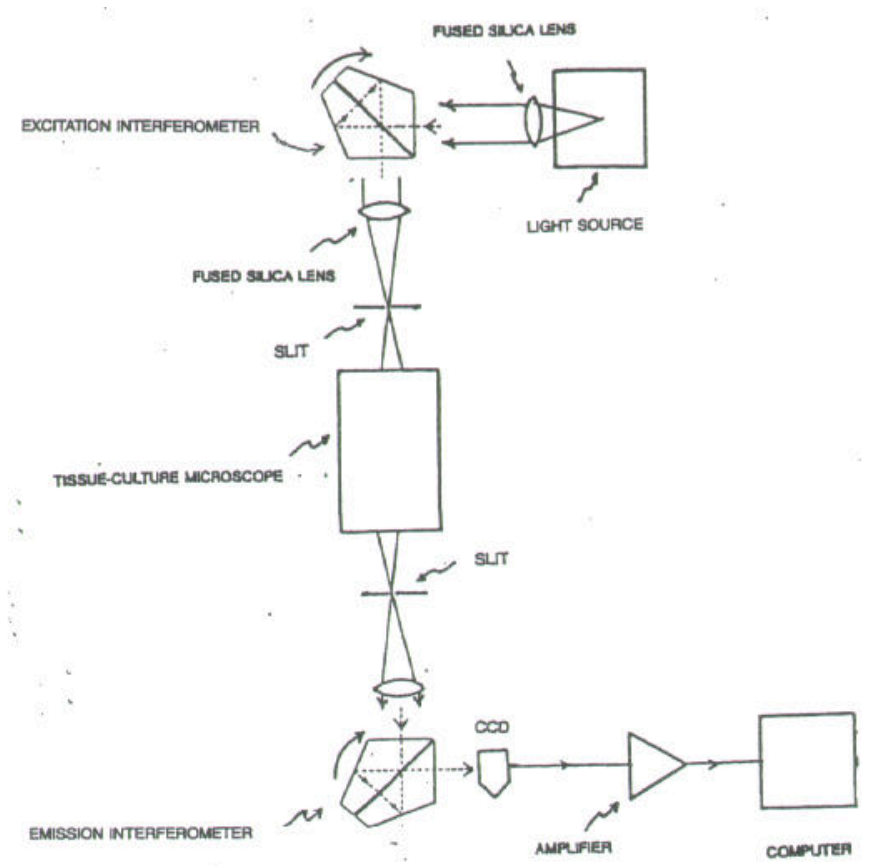

Figure 3: Microscope equipped with Pentaferometer on excitation and emission sides used for spectral fluorescence imaging. 